\title{
Diurnal Variation of Cosmic Ray Intensity at Different Group of Geomagnetic Index (Ap)
}

\author{
Divya Dubey ${ }^{1}$, Suryanshu Chudhary ${ }^{1}$, Ambika Singh ${ }^{2}$, Anil Kumar Tiwari ${ }^{3}$ \\ ${ }^{1}$ Physics Department, AISECT University, Bhopal (Madhay Pradesh), India \\ ${ }^{2}$ Physics Department, NRI Institute of Information Science and Technology, Bhopal (Madhay Pradesh), India \\ ${ }^{3}$ Physics Department, Govt. T. R. S. College, Rewa (Madhay Pradesh), India
}

\section{Email address:}

msc_divya86@yahoo.in (D. Dubey),csuryansh@gmail.com (S. Chudhary), ambika.physics@gmail.com (A. Singh), tiwarianil_trs@rediffmail.com (A. K. Tiwari)

\section{To cite this article:}

Divya Dubey, Ambika Singh, Suryanshu Chudhary, Anil Kumar Tiwari. Diurnal Variation of Cosmic Ray Intensity at Different Group of Geomagnetic Index (Ap). International Journal of Astrophysics and Space Science. Vol. 5, No. 4, 2017, pp. 63-70. doi: 10.11648/j.ijass.20170504.12

Received: September 1, 2017; Accepted: September 22, 2017; Published: November 6, 2017

\begin{abstract}
The solar modulation of Galactic Cosmic Rays (GCR) is revealed in the record of neutron monitors in terms of daily variation. Day-to-day and long term basis daily variations have been investigated for the recent period from 1965 to 2015 along with geomagnetic disruption index Ap on twelve-monthly average basis. Here Ap index has been used as a placeholder for the solar bring on interplanetary disturbances. It is create that, in general on an annual average basis, both the diurnal amplitude as well as the diurnal phase is considerably lower in the years of low Ap values, through the period 1965 to 2015 . Such as a result was also informed for the earlier periods. However, anomalous and determined results for the diurnal variations have been received when the daily values of Ap are diffused in five groups with the first group demonstrating low Ap condition (i.e. quite periods) and the last group naturally representing the disturbed conditions.
\end{abstract}

Keywords: Solar Activity, Cosmic Ray, Geomagnetic Index, Diurnal Amplitude, Diurnal Phase

\section{Introduction}

The spatial property anisotropy of the galactic cosmic radioactivity in the interplanetary medium is mentioned with daily variation of cosmic ray intensity by using milled based detectors. The detector on earth scans the whole spiritual sky during a period of 24 hours. The earth finishes one revolution around its own axis, once in 24 hours (a solar day). Many scientists are using the data of milled based detectors, have been informed Excess of particles incoming from the asymptotic direction around 18 hours local time. The amplitudes and phases of the harmonic mechanisms of the daily variation of cosmic rays are in the main derivative by Fourier analysis and using the hourly counts of neutron and meson monitors. A large dissimilarity in either the daily amplitude or in the time of highest between different, but virtually stations are symptomatic that the anisotropy is fast changing within 24 hours, or the universal time effects are predominant. The solar diurnal variation of the cosmic-ray intensity interpreted initially on the basis of outward radial convection due to magnetized supersonic solar wind and an inward dissemination along the interplanetary magnetic field. The stability among the convection and circulation generates energy which is independent anisotropic flow of cosmic-ray particles from the 18 hours co-rotational direction. The polarity state is defined as "negative" ("positive") when the solar magnetic field is directed inward (outward) from the sun in the northern Hemisphere and outward (inward) in the southern Hemisphere. The magnitude and direction of the anisotropic cosmic ray flow along with its variant with an 11 year sunspot cycle and 22 year solar magnetic cycle can provide vital observational constraints for analysis the principles of the carrying solar modulation of galactic cosmic rays. Many models are sensitive to the route of the sun's magnetic field. The drift models prophesy that there should be a radiating gradient of cosmic ray density with a larger value in epochs of negative solar magnetic polarity than in epochs of positive solar polarity. Models also predicted a bidirectional latitude gradient that a reverses its sign with the 
reversal of the solar magnetic field [1-5]. The performance of the diurnal time of supreme has been shown regular with the convective-diffusive instrument. That mechanism relates the solar diurnal anisotropy of cosmic rays to the dynamics of the solar wind and interplanetary magnetic field. The fieldaligned directions of the diffusive vector, independent of the interplanetary magnetic field polarity, have been confirmed [6]. In general, the solar diurnal anisotropy is caused by the modulation of the galactic cosmic rays in the heliosphere [7], [8]. Cosmic rays enter into heliosphere and gyrate along the interplanetary magnetic field lines. Magnetic flux indiscretions scattered cosmic rays from gyro-orbits which are initiating diffusion. The solar wind also convects cosmic rays outward while particles wayfaring along a regular portion of magnetic field a line which undergoes magnetic curvature and drift motion. Long-term medians of the solar diurnal variation deliver information about the average behavior of cosmic rays in the vicinity of the earth. From the time when the diurnal anisotropy is caused by the solar modulation which can use the observed effect to derive the information about the underlying solar modulation processes [9], [10]. The results of neutron monitor observations from earlier periods shown that the average characteristics of the diurnal anisotropy has been generally invariant through the period 1958-70, neutron monitor energies reasonable in terms of the convection-diffusion theory [11], [8]. However, later on observations have shown that the average characteristics of the diurnal anisotropy varied significantly during the period of 1971-74 and that was particularly evident from the detailed studies reported [12]. The energy dependent phase shift to earlier hours in the diurnal time of maximum of the cosmic ray intensity was first pointed out in the year 1971 [13]. These new results, the data of low latitude stations (high cutoff rigidity) are very crucial [14]. Observed diurnal anisotropy is related to sunspot cycle, which also shown rigidity dependent. Moreover, the amplitude and phase of the diurnal anisotropy changes with period of one or two solar cycles [11-12], [14-22]. The time of the maximum diurnal variation shows remarkable and systematic phase shift towards earlier hours compare than normal. According to the modern theory, the convection, diffusion, drift and energy change of the cosmic ray subdivisions in the separated magnetized solar wind are overall methods and mainly liable for the modulation of galactic cosmic rays (GCR).

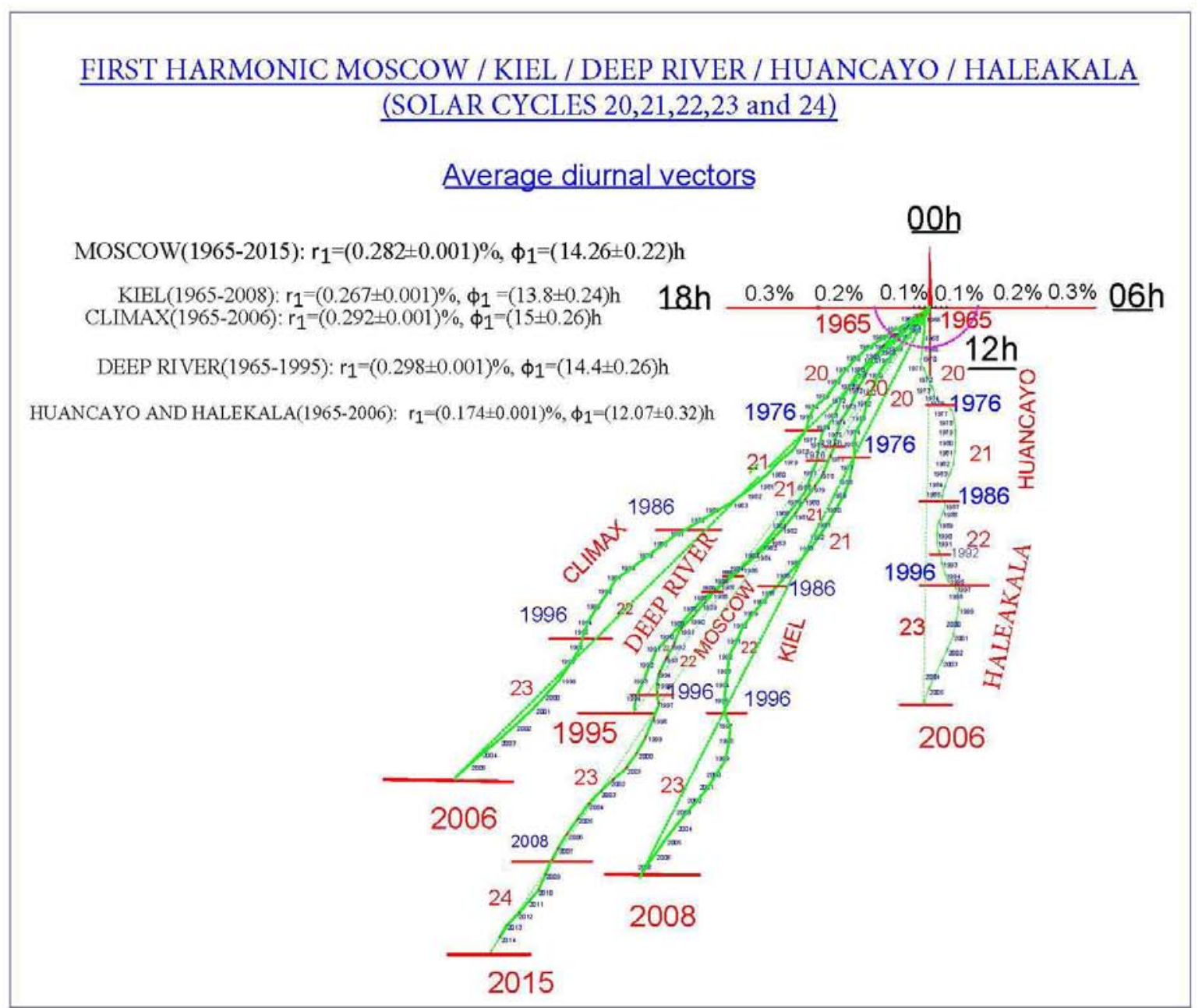

Figure 1. The Vector-addition diagram of the annual-average vectors of the first harmonic of the observed daily variation of cosmic rays of Moscow neutron monitor station for the years 1965-2015, Kiel neutron monitor station for the years 1965-2008, Deep River neutron monitor station for the years 1965-1995, Climax neutron monitor station for the years 1965-2006 and Huancayo neutron monitor station for the years $1965-1990$ and Halekala neutron monitor station for the years 1991-2006. The overall average values are also marked. 


\section{Data Analysis}

Present analysis has been performed by collected the records of diurnal amplitudes and phase of cosmic ray anisotropies for the period 1965-2015 of Moscow neutron monitor station and for the period 1965-2008 of Kiel neutron monitor station (high-latitude station) and for the period 1965-2006 of Climax neutron monitor station and for 1965-2006 of Huancayo and Haleakala neutron monitor stations (low latitude). Also correlated with twelve-monthly average basis geomagnetic index (Ap) covering the previous solar cycles $20,21,22,23$ and present solar cycle 24(1965-2015). The pressure corrected hourly neutron monitor data from super neutron monitor stations from Moscow $\left(55.4^{\circ} \mathrm{N}, 37.3^{\circ} \mathrm{E}, 2.39 \mathrm{GV}\right)$, Kiel $\left(54.3^{\circ} \mathrm{N}, 10.1^{\circ} \mathrm{E}, 2.36 \mathrm{GV}\right)$, Climax $\left(39.3^{\circ} \mathrm{N}, 106.1^{\circ} \mathrm{W}\right.$, $2.97 \mathrm{GV})$, Huancayo $\left(12.03^{\circ} \mathrm{S}, 75.35^{\circ} \mathrm{W}, 12.92 \mathrm{GV}\right)$ and Haleakala $\left(20.8^{\circ} \mathrm{N}, 156.2^{\circ} \mathrm{W}, 13.3 \mathrm{GV}\right)$ have been observed for this study. These three stations consist of high and low cutoff rigidities, which respond to different energy range of cosmic ray particles. The pressure-corrected high counting-rate neutron monitor data having data different cut off rigidities, harmonic analysis to derive amplitude (\%) and phase (hours), for period of 51 years (1965-2015). The observed diurnal anisotropy (first harmonic of daily variation) vectors in local time have been examined by plotting them on a vector addition diagram, after classifying, correlation between amplitude and geomagnetic index Ap.

\section{Discussion}

Each day vectors of the daily cosmic rays variations are vectorially added to provide the average values including the annual average. To monitor the vectorial changes from one year to another, it is useful to plot the values in vector form. In fact, these averages are generally studied by depicting them all as a harmonic dial illustration and in vector addition format. Figure 1 shows the vector addition diagram of annual average vectors of the first harmonic of the daily variation of cosmic rays from Moscow neutron monitor station for the years 1965-2015, Kiel neutron 'monitor station for the years 1965-2008, Deep River neutron monitor station for the years 1965-1995, Climax neutron monitor station for the years 1965-2006, Huancayo and Haleakala neutron monitor station for the years (1965-2006), from this figure, it is very clearly noticed that the diurnal phase has continuously shifting to earlier and later hours in various phases of solar cycles. The overall average diurnal vectors for the Kiel, Moscow, Deep River, Climax, Tokyo, Huancayo and Haleakala neutron monitor stations have been deduced.

Moscow (1965-2015): $\mathrm{r}_{1}=(0.282 \pm 0.00087) \%, \quad \phi_{1}=$ $(14.26 \pm 0.22) \mathrm{h}$

Kiel (1965-2008): $\mathrm{r}_{1}=(0.267 \pm 0.00090) \%, \phi_{1}=(13.8 \pm 0.24) \mathrm{h}$

Climax (1965-2006): $\mathrm{r}_{1}=(0.292 \pm 0.0010) \%, \phi_{1}=(15 \pm 0.26) \mathrm{h}$

Deep River (1965-1995): $\mathrm{r}_{1}=(0.298 \pm 0.0011) \%, \phi_{1}=$ $(14.4 \pm 0.26) \mathrm{h}$

Huancayo and Haleakala (1965-2006): $r_{1}=(0.174 \pm 0.0015) \%$, $\phi_{1}=(12.07 \pm 0.32) \mathrm{h}$

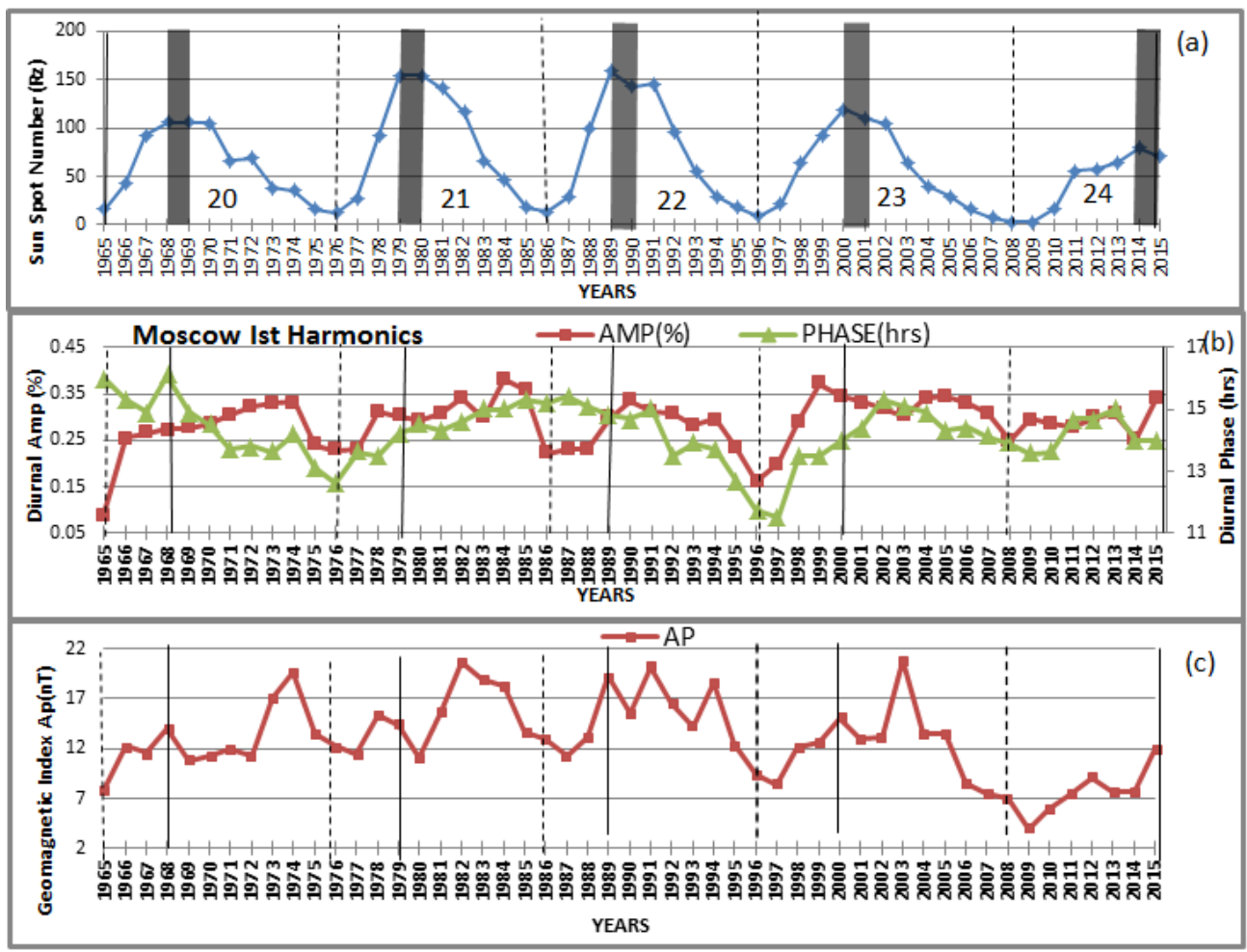

Figure 2. Shows the yearly averages of (a) Sunspots number (b) Diurnal vectors (amp. and phase for Moscow Neutron Monitor) (c) Geo-magnetic index (Ap) for each year from 1965 to 2015 (solar cycle 20 to 24). 


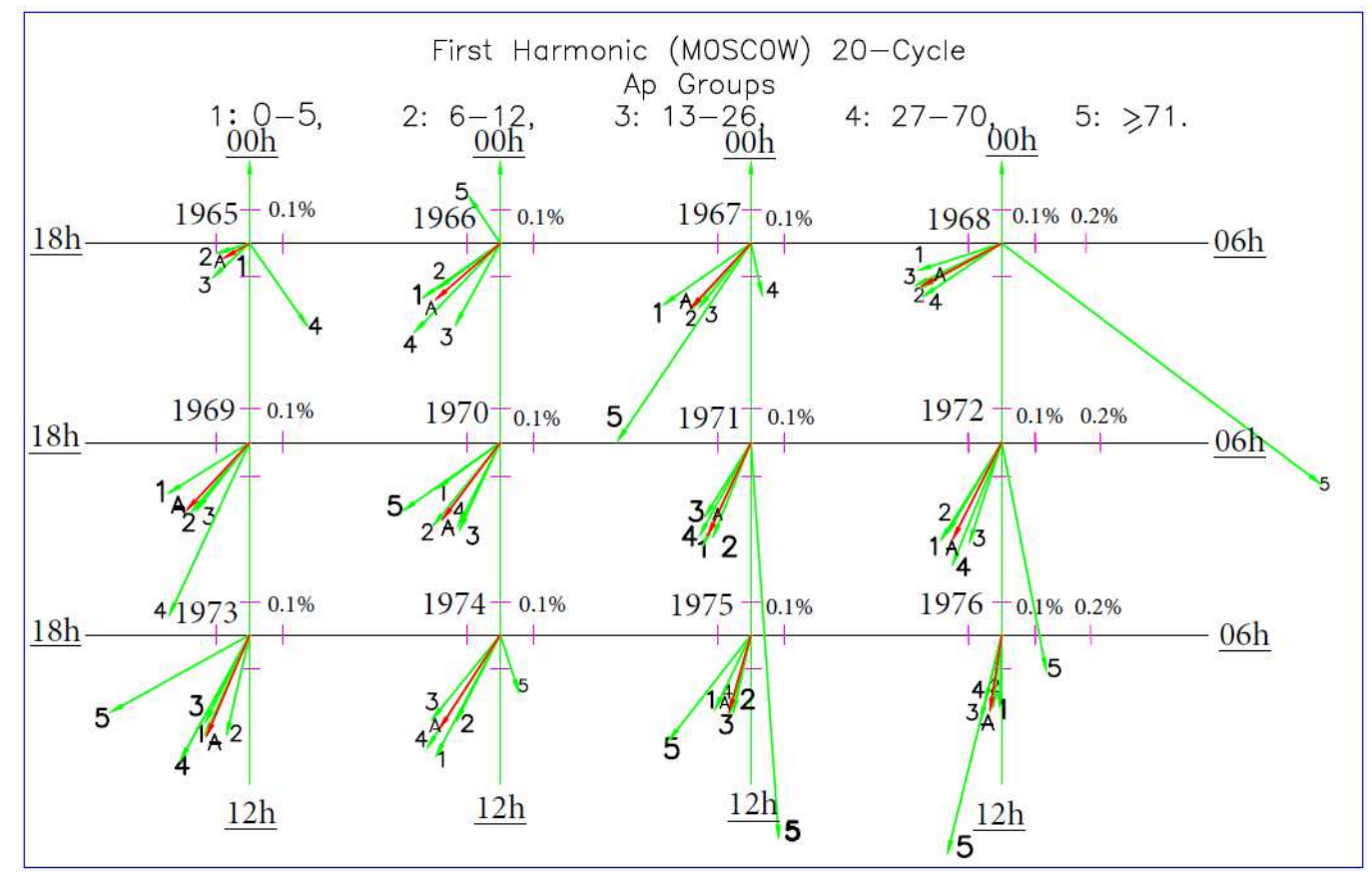

Figure 3. Shows the average diurnal vectors for the years 1965-1976 (solar cycle 20) for various Ap group, for station Moscow, (low cut-off rigidity).

The long term vector addition diagram of annual average vectors of first harmonic of diurnal variation it is clearly notice that the annual average diurnal phase is shifting to before hours during the nearly solar activity minima in all the even solar cycles (cycles 20,22). It is observed to move in later hours during the odd solar cycles $(21,23)$. Figure 2 shows the annual average values of diurnal vectors (amplitude and phase) of Moscow neutron monitor station, geomagnetic index (Ap), sunspot number (R). The geomagnetic index (Ap) is peak during the abating phase of solar cycles, it is reached the highest value in 2003 and the deepest value in 2009. Figure 2 shows the annual average diurnal amplitude is generally maximum near the solar activity maxima and in its declining phase. The amplitude of diurnal vectors varies with a period of one sunspot cycle (11year) while the phase of diurnal vectors varies with solar magnetic cycles (22-year cycle). Where the Years of solar activity minimum and maxima for each cycle are also marked by vertical lines (dashed lines and full lines respectively). Moscow neutron monitor data have been used to drive the daily vector of cosmic ray anisotropy observed days are divided into five groups according to increasing value of Ap index (0-5, 6-12, 13-26, 27-70 and $\geq 71)$. Higher Ap groups are indicators of more disturbed interplanetary medium. In figure 3 , shows that during the period of high sunspot activity of solar cycle 20 (1967-1971), the diurnal vector rotates anticlockwise only when Ap is high but diurnal phase is small, contrary to that observed on long-term basis shown earlier by considering the annual averages. Nevertheless, during the declining phase of solar activity, including the minima (1973-1976), the diurnal vector rotates clockwise with the increase in the value of Ap index. Moreover, in general, the diurnal phase is shifted to earlier hours during the period 1973 to 1976 (A>0). For cycle 21, the period of high sunspot activity (1979-1981), the diurnal vector rotates anticlockwise shown in figure 4, i.e. when Ap is high the diurnal phase is smaller, again contrary to that observed on long-term basis shown earlier by considering the annual averages; such a trend continues, during the declining phase of the solar activity (1984-86) also, where again the diurnal vector rotates anticlockwise with the increase in the value of Ap index. For cycle 22, in figure 5, clearly observed that in the years 1986, 1988, 1990 and 1991 the diurnal vector rotates anticlockwise, i.e. when Ap is high the diurnal phase is smaller, whereas, during the declining phase of solar activity (1991-1996), the diurnal vector rotates clockwise with the increase in the value of Ap index, which is in according with the findings on annual average basis. Moreover, in general the diurnal phase is shifted to earlier hours in the years 1994-1996 (A>0). For cycle 23 shown in figure 6 , we observed that in the early yearly i.e. up to year 2000 , the rotation of the diurnal vector in very small. But quite often it is tending to be clockwise; however the change is insignificant from the year 2001 onwards. The diurnal vectors are also seen significantly rotate anticlockwise, covering the whole period of the declining phase of solar activity, including in minima. For solar cycle 24 shown in figure 7 , we observed that during the period of high sunspot acting of solar cycle 24, the diurnal vector are rotates anticlockwise i.e. when Ap is high the diurnal phase is smaller. The diurnal amplitudes and phase are minimum during the periods (2008-10) of solar cycle 24 . The value of Ap is very low during the periods 2008, 2009 and 2010. As such, the results are found to be a little different from other solar cycle. Nevertheless, in conclusion, we can say that the diurnal vectors rotate in the anticlockwise direction in the cycle 23 also, as was the case in cycle 21 . Finally we conclude that the diurnal vector rotates anticlockwise during 
the declining phase of odd solar cycle $(21,23)$. However, it rotates clockwise during the declining phase (near minima) of even solar cycles (20 and 22). Moreover, the diurnal phase is found to be shifted to earlier hours only in these two cycles 20 and 22. It is also found that in the observed diurnal anisotropy for low Ap groups (1 to 3 ) the dispersion is large for high Ap values groups (4 to 5). The large variability during the Ap days and the significant increase in the diurnal amplitude with increase in Ap values use also observes in other neutron monitor. Figure 8 shows the scatter-plot of the annual average of diurnal amplitude and geomagnetic index (Ap) for all the 51 years from 1965-2015 positive correlation $(\mathrm{r}=0.4 \pm \ldots .$.$) is evident. The positive correlation means that$ when the diurnal amplitude is larger, the geomagnetic index (Ap) is also larger. Cross-correlation between the annual average of geomagnetic index (Ap) and phase of diurnal variation of cosmic ray intensity for the period 1965-2015 have been plotted in figure 9. From this figure it is clear that no correlation between geomagnetic index (Ap) and diurnal amplitude of cosmic ray intensity.

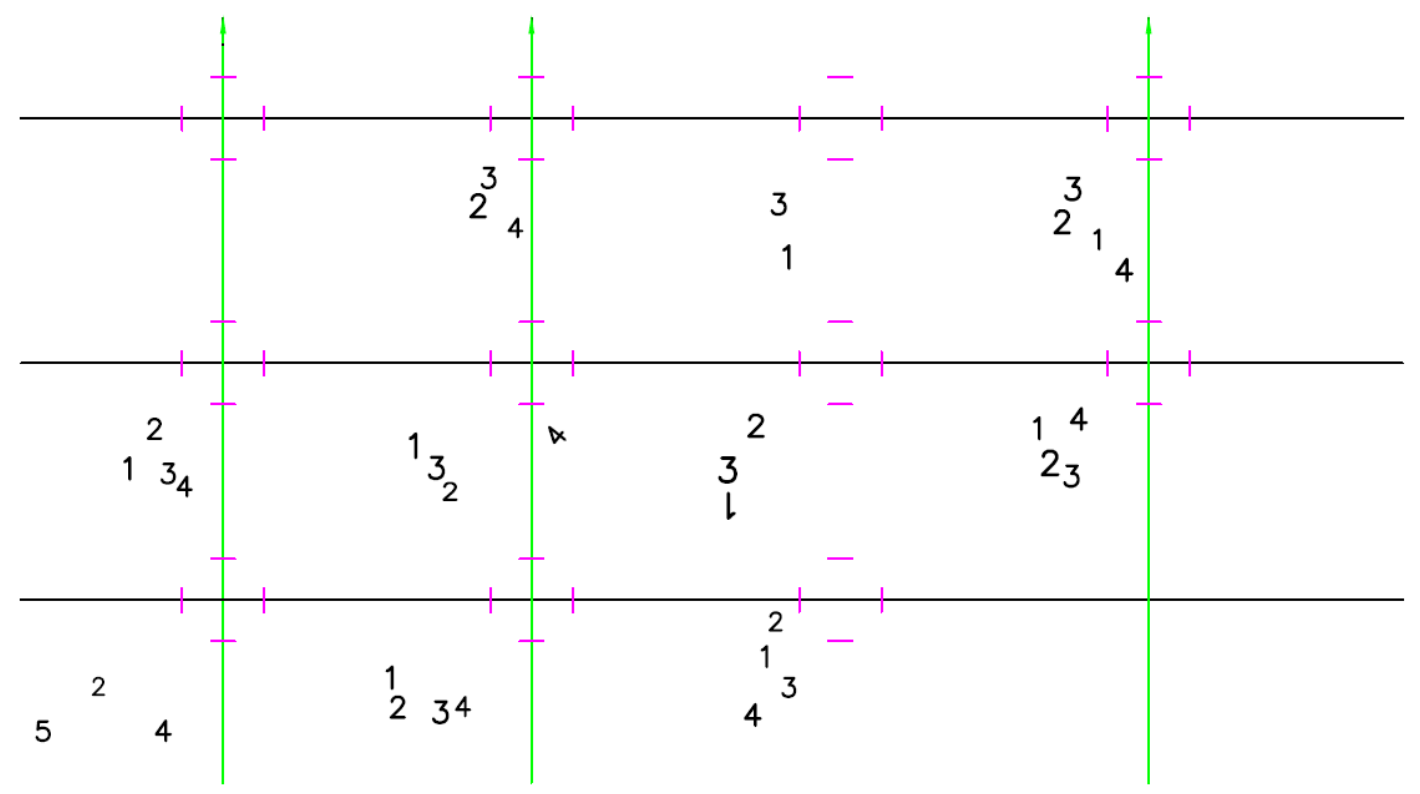

Figure 4. Shows the average diurnal vectors for the years 1976-1986 (solar cycle 21) for various Ap group, for station Moscow, (low cut-off rigidity).

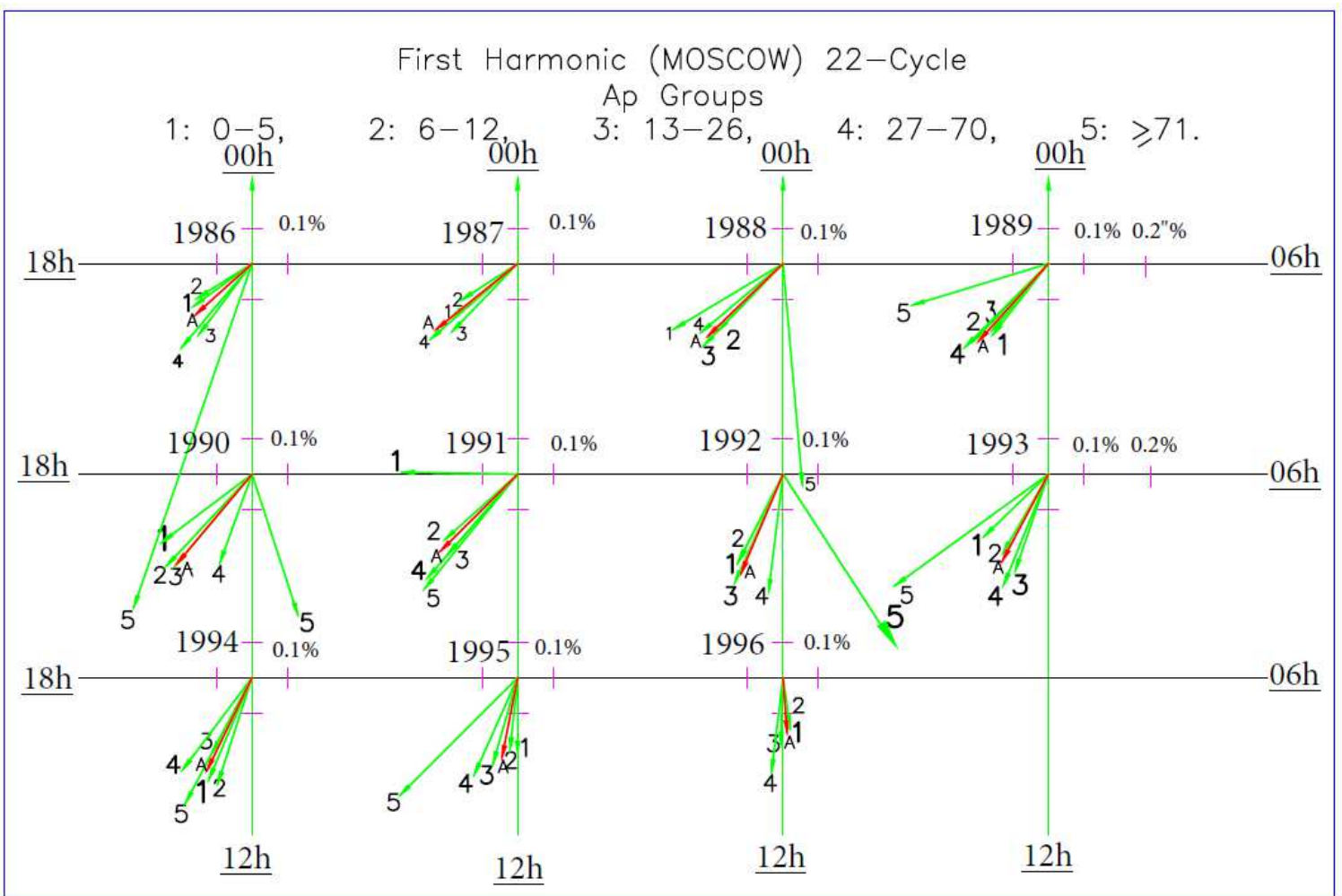

Figure 5. Shows the average diurnal vectors for the years 1986-1996 (solar cycle 22) for various Ap group, for station Moscow, (low cut-off rigidity). 


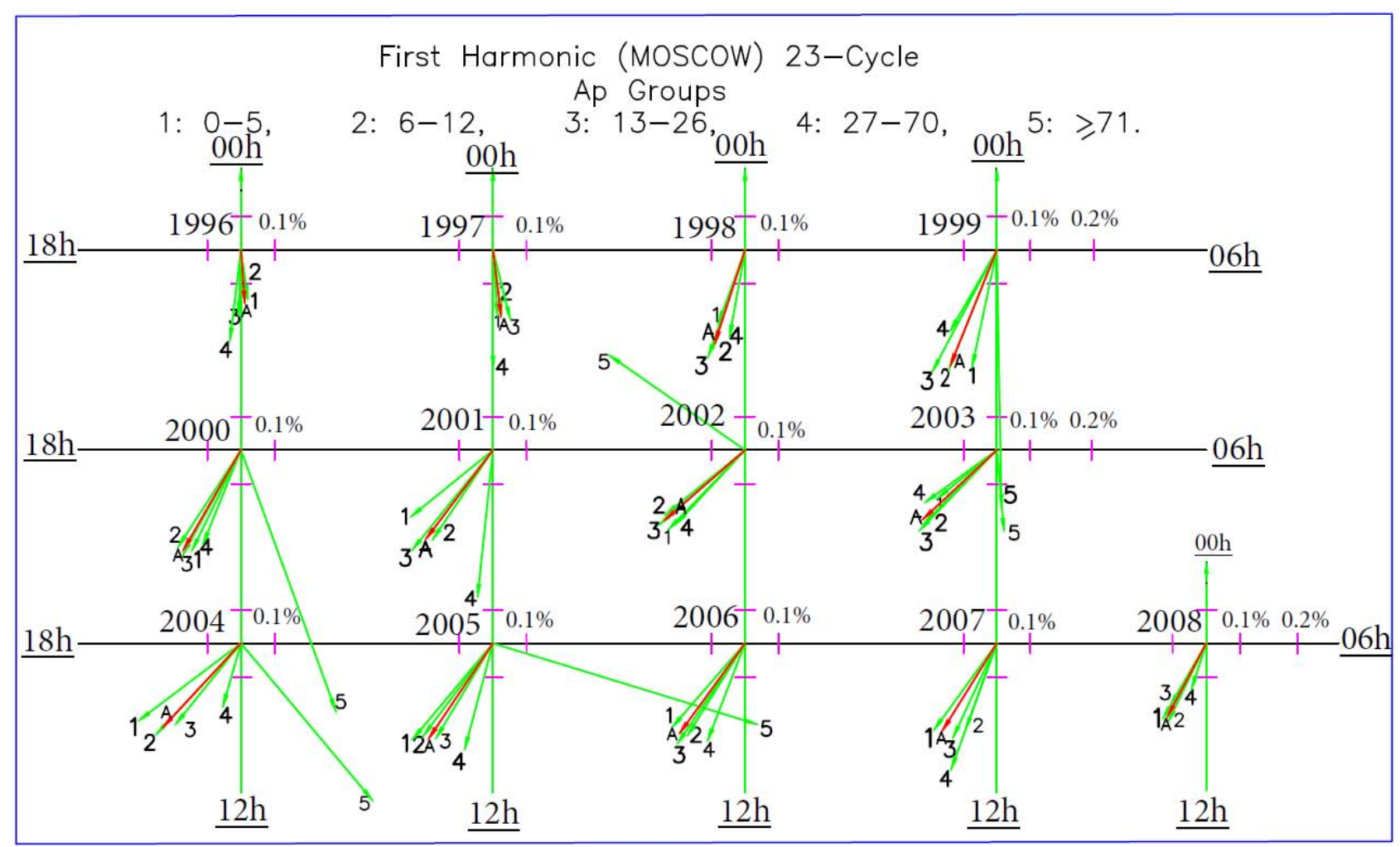

Figure 6. Shows the average diurnal vectors for the years 1996-2008 (solar cycle 23) for various Ap group, for station Moscow, (low cut-off rigidity).

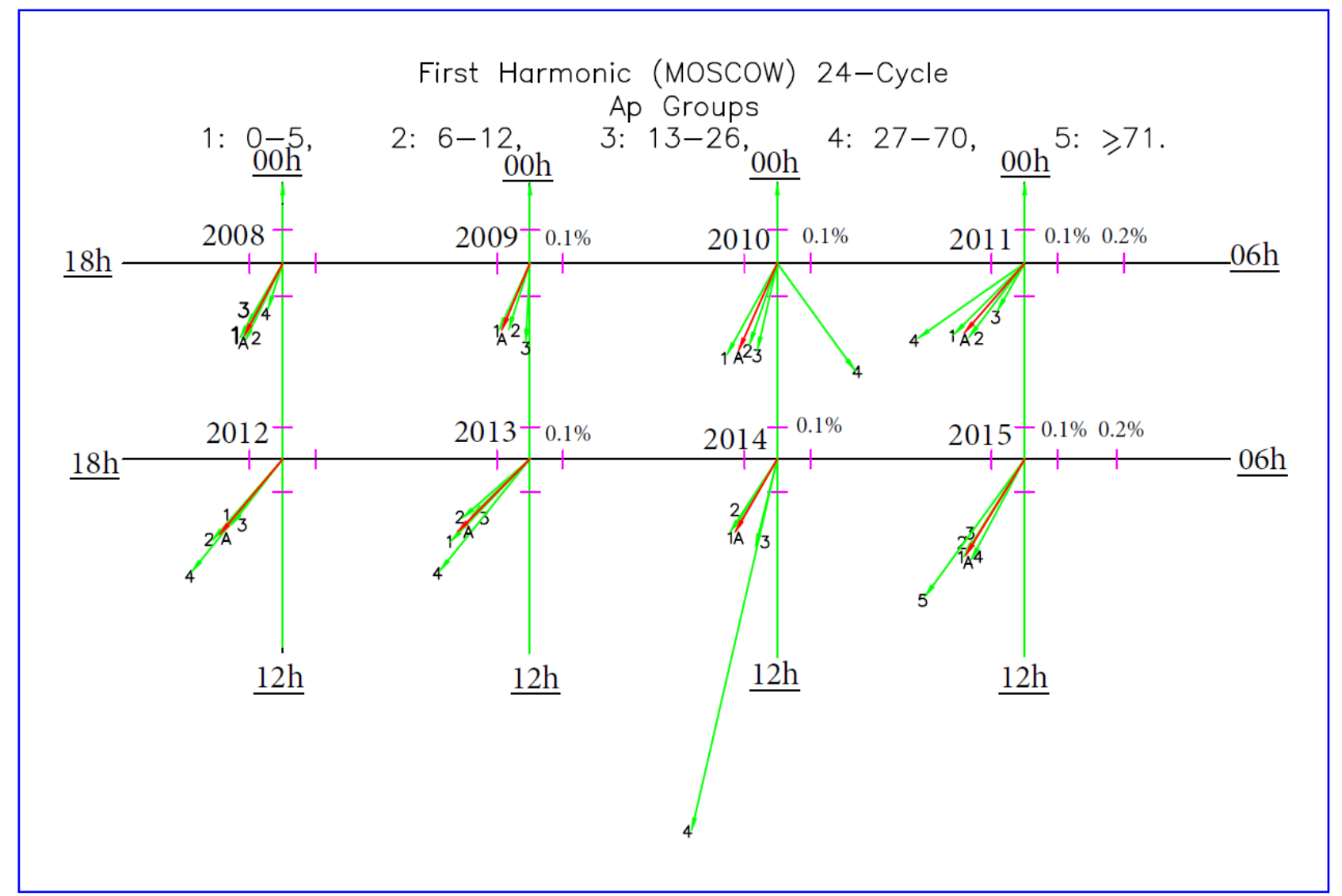

Figure 7. Shows the average diurnal vectors for the years 2008-2015 (semi-solar cycle 24) for various Ap group, for station Moscow, (low cut-off rigidity). 


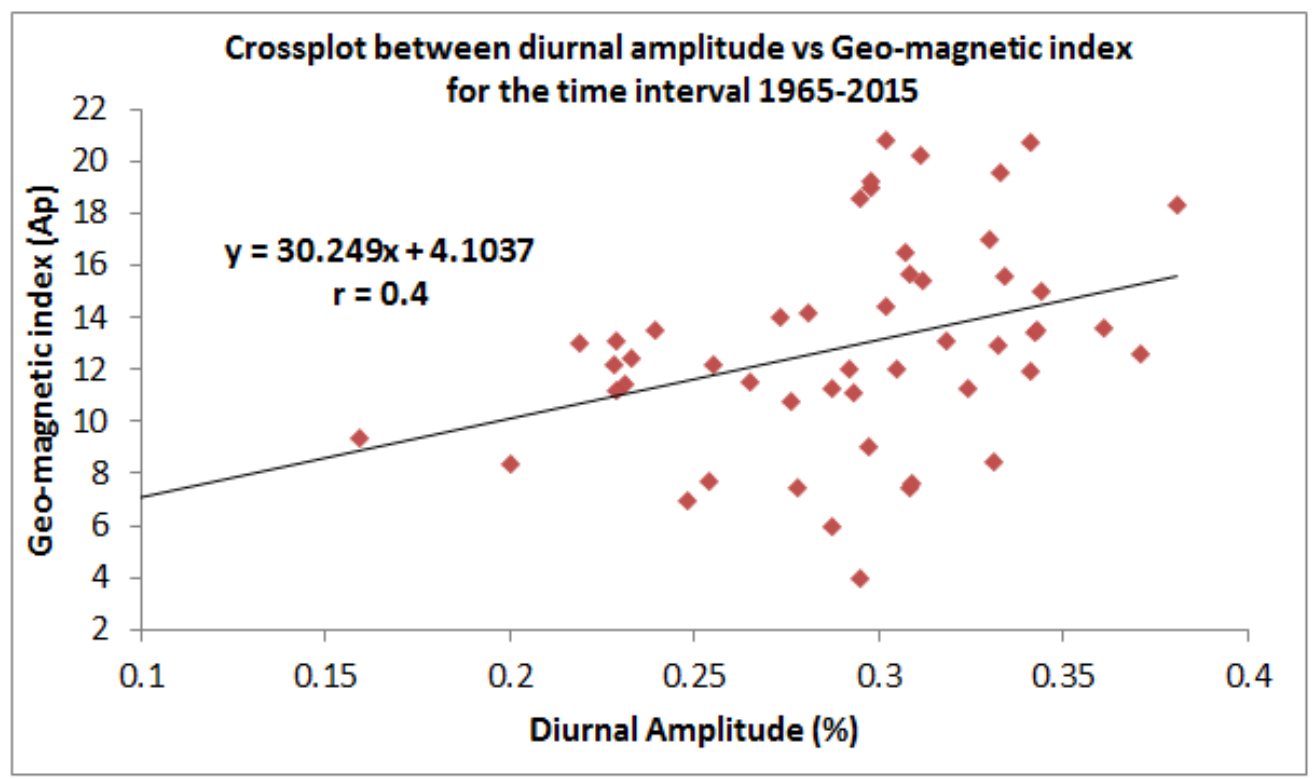

Figure 8. Show the cross plot between annual average of diurnal amplitudes of cosmic ray intensity of Moscow neutron monitor and geomagnetic index Ap for the time interval 1965-2015.

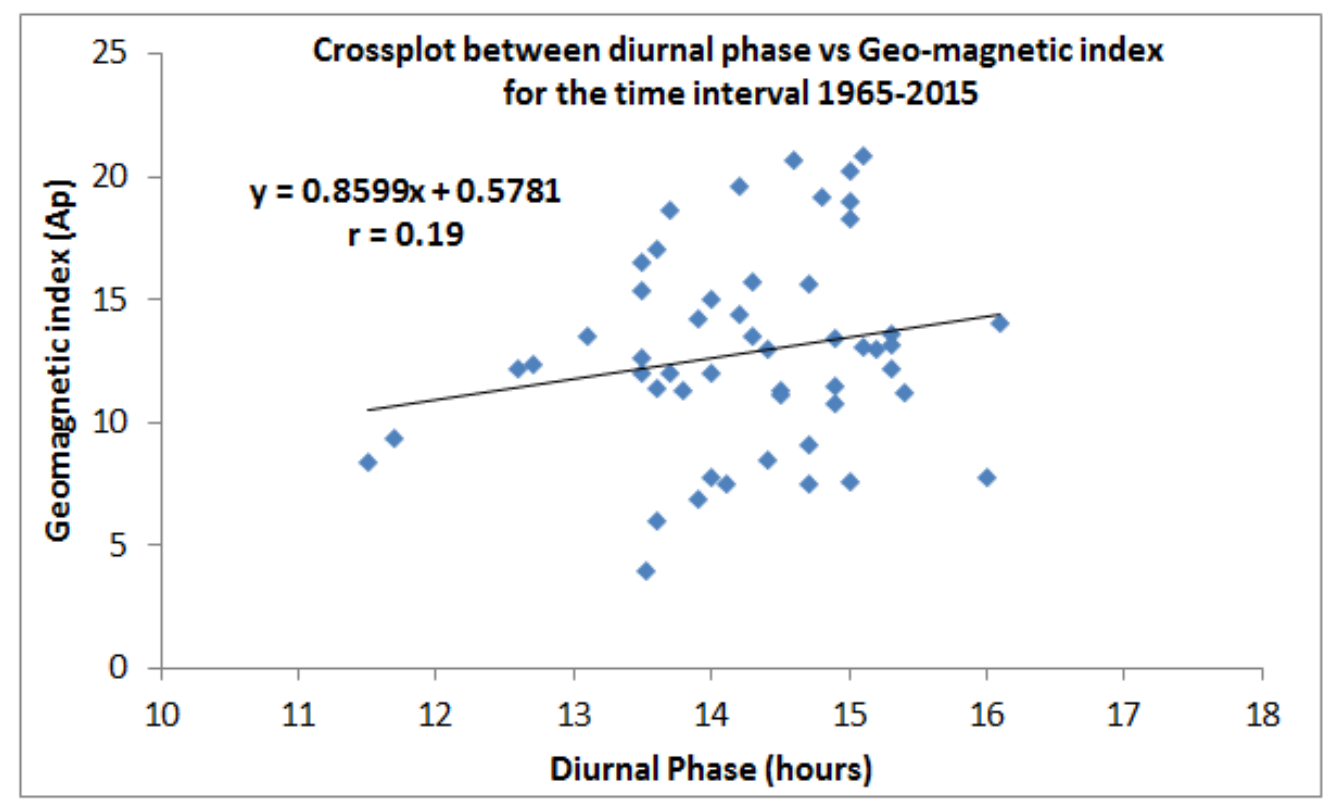

Figure 9. Show the cross plot between annual average of diurnal phase of cosmic ray intensity of Moscow neutron monitor and geomagnetic index Ap for the time interval 1965-2015.

\section{Conclusions}

a. The analysis has also been performed on day-to-day basis for each year using the daily vectors for Moscow station by dividing them into five groups in each case. In most of the years, it is observed that normally the average diurnal vectors for groups representing more disturbed periods in geomagnetic disturbance index Ap and the continuously low Ap days. The shift of average phase in the anticlockwise direction and the amplitude is relatively larger. Nevertheless, a clear cut distinctly different effect has been noticed for the period of minimum activity and 2 to 3 years before the minimum activity period (i.e. in the final declining phase of the solar activity).

b. The long-term vector addition diagram of annual average vectors of the first harmonic of the daily variation, it is clearly noticed that the annual average diurnal phase is continuously shifting to earlier hours up to the solar activity minima in all the even solar cycles. Whereas, it is observed to shift back in later hours during odd solar cycles.

c. A further peculiarity is noticed that during the even solar cycles the annual average diurnal amplitude is generally maximum near the solar activity maxima and 
in its declining phase. In contrast, during the odd solar cycles the diurnal amplitude is generally maximum only near the minimum solar activity.

d. The amplitude of the cosmic ray diurnal variation measured by ground based detectors varies mostly with a period of one sunspot $(\sim 11$ years $)$ cycle. While the phase of the diurnal variation has a small component with a period of one solar cycle. The dominant component varies with two sunspot cycles $(\sim 22$ year cycle $)$.

\section{Acknowledgements}

We are thankful and acknowledged to NSSDC and OMNI database centers for providing the data as geomagnetic index and neutron monitor network. This online facility gives help us to make meaningful conclusion.

\section{References}

[1] Jokipii, J. R. and Kopriva, D. A.; "Effects of particle drift on the transport of cosmic rays, III, Numerical models of galactic cosmic-rays", Astrophysics Journal, 234, 384(1979).

[2] Jokipii, J. R. and Davila, J. M.; "Effects of particle drift on the transport of cosmic rays, IV, More realistic diffusion coefficients", Astrophysics Journal, 248, 1156-1161(1981).

[3] Potgieter, M. S. and Moraal, H.; "A drift modal for the modulation of cosmic rays", Astrophysics Journal, 294, 425440 (1985).

[4] Jokipii, J. R. and Kota, J.; "Cosmic rays near the heliospheric current sheet, 2 . An ensemble approach to comparing theory and observation", Journal Geophysics Research, 91, 28852888 (1986).

[5] Potgieter, M. S., Le Roux. J. A., Burger, R. A.: 1989, Journal Geophysics Research. 94, 2323.

[6] Mavromichalaki, H.; "Application of diffusion convection model to diurnal anisotropy data", Earth, Moon and Planets, 47, 61-72 (1989).

[7] Parker, E. N.; "Theory of streaming of cosmic rays and the diurnal variation", Planetary Space Science, 12, 735-749 (1964).

[8] Forman, M. A. and Gleeson, L. J.; "Cosmic ray streaming and anisotropies" Astrophysics Space Science, 32, 77-94 (1975).

[9] Venkatesan, D. and Badruddin; "Cosmic ray intensity variations in the 3-dimentional heliosphere" Space Science Reviews, 52, 121-194 (1990).
[10] Hall, D. L., Duldig, M. L. and Humble, J. E.; "Analysis of sidereal and solar anisotropies in cosmic rays", Space Science Reviews, 78, 401-442 (1996).

[11] Rao, U. R.; "Solar modulation of galactic cosmic radiation", Space Science Reviews., 12, 719-809 (1972).

[12] Agrawal, S. P. and Singh, R. L.; "Critical study of the diurnal and semi-diurnal variation of cosmic ray intensity on day-today basis", Proceedings $14^{\text {th }}$ International Cosmic Ray Conference., Munich (West Germany), 3, 1253-1257 (1975).

[13] Agrawal, S. P. and Ananth, A. G.; "Average characteristics of cosmic ray diurnal anisotropy at relativistic energies", Proceedings $13^{\text {th }}$ International Cosmic Ray Conference, Denver (USA), 2, 1005-1010 (1973).

[14] Agrawal, S. P. and Bercovitch, M.; "Study of 11, 22 year periodic variation of cosmic ray diurnal anisotropy", Proceedings $18^{\text {th }}$ International Cosmic Ray Conference, Bangalore (India), 3, 316-319 (1983).

[15] Pomerantz, M. A. and Duggal, S. P.; "The cosmic ray solar diurnal anisotropy", Space Science Reviews 12, 75-130 (1971).

[16] Ahluwalia, H. S.; "Cosmic ray modulation near the onset and maximum phases of solar activity cycle 22", Planetary Space Science, 40, 1227 (1996).

[17] Belov, A. V., Eroshenko, E. A., Oleneva, V. A. and Yanke, V. G.; "Relation the Forbush effects to the interplanetary and geomagnetic parameters", Proceedings $27^{\text {th }}$ International Cosmic Ray Conference, Hamburg (Germany), SH 2.1, 9, 3552 (2001).

[18] Tiwari, C. M., Agrawal, S. P., Tiwari, D. P., El-Borie, M. A., Shrivastava, P. K.; "Study of daily variation of cosmic ray intensity for the period 1989-2000", Journal Current Science, 3(1), 219-222 (2003).

[19] Singh, Y. P. and Badruddin; "Statistical consideration in superposed epoch analysis and its applications in space research", Journal Atmospheric Solar Terrestrial Physics, doi:10.1016/J.jastp.2006. 01.007 (2006).

[20] Singh, Ambika, Tiwari, Anil Kumar and Agrawal, S. P.; "Study of High and Low Amplitude Wave Trains of Cosmic Ray Diurnal Variation during solar cycle 23", Journal Astrophysics Astronomy, 31, 89-96 (2010).

[21] Tiwari, Anil Kumar, Singh, Ambika, Agrawal, S. P; "Study of the diurnal variation of cosmic rays during different phases of solar activity", solar physics, 279, 253-267 (2012).

[22] Singh, Ambika, Dubey, Divya, Singh, Rukmani Pratap, Tiwari, Anil Kumar; "Variation of Upper Cut-off Rigidity of Cosmic Ray Diurnal Anisotropy", International Journal Innovative Research Science Engineering Technology, Vol. 2, ISSUE 9, 4648-4654 (2013). 